BULLETIN Bulletin hispanique

HISPANIQUE Université Michel de Montaigne Bordeaux

$115-2$ | 2013

Les traductions vieillissent-elles?

\title{
Reflexiones sobre la práctica colectiva de la traducción
}

Algunas versiones de poesía catalana y francesa moderna

\section{Clara Curell}

\section{OpenEdition}

\section{Journals}

Edición electrónica

URL: http://journals.openedition.org/bulletinhispanique/2808

DOI: 10.4000/bulletinhispanique.2808

ISSN: 1775-3821

\section{Editor}

Presses universitaires de Bordeaux

\section{Edición impresa}

Fecha de publicación: 28 diciembre 2013

Paginación: 637-651

ISBN: 978-2-86781-908-7

ISSN: 0007-4640

Referencia electrónica

Clara Curell, «Reflexiones sobre la práctica colectiva de la traducción », Bulletin hispanique [En línea], 115-2 | 2013, Publicado el 05 enero 2017, consultado el 24 abril 2019. URL : http://

journals.openedition.org/bulletinhispanique/2808 ; DOI : 10.4000/bulletinhispanique.2808 


\title{
Reflexiones sobre la práctica colectiva de la traducción: algunas versiones de poesía catalana y francesa moderna
}

\author{
Clara Curell \\ Universidad de La Laguna
}

La conception traditionnelle de la traduction d'un texte poétique, envisagé comme démarche individuelle s'est trouvée enrichie ces derniers temps par une pratique collective qui s'est révélée très satisfaisante et qui est la garantie d'un travail bien fait. Dans cet article, nous analysons les procédés et les critères les plus importants qui sont utilisés au sein $d u$ «Taller de Traducción Literaria de la Universidad de La Laguna», et nous les illustrons d'exemples tirés de versions récentes de poèmes catalans et français.

Mots-clés : traduction collective, Pierre Reverdy, Joan Carner, Taller de Traducción Literaria de la Universidad de La Laguna.

En los últimos años, al método tradicional de enfrentarse a la traducción de un texto poético de forma individual se ha sumado una modalidad especialmente satisfactoria y que garantiza un trabajo bien hecho, como es la traducción colectiva. En este artículo, analizamos los principales procedimientos y criterios que seguimos en el Taller de Traducción Literaria de la Universidad de La Laguna, que ilustramos con ejemplos de versiones recientes de poemas catalanes y franceses.

Palabras claves: traducción colectiva, Pierre Reverdy, Joan Carner, Taller de Traducción Literaria de la Universidad de La Laguna.

In addition to the traditional individual approach to the translation of poetic texts, recent years have seen a move towards collaborative translation, an approach that proved extremely satisfactory, and also guarantees that the finished product is of high quality. In this paper, we analyse the main procedures followed and the criteria employed by the "Taller de Traducción Literaria de la Universidad de La Laguna", illustrating our account with examples drawn from recent translations of Catalan and French poetry.

Keywords: Collective translation, Pierre Reverdy, Joan Carner, Taller de Traducción Literaria de la Universidad de La Laguna. 
$\mathrm{F}$ rente a las numerosas negaciones de la viabilidad de la traducción poética ${ }^{1}$ se alza el hecho de su ininterrumpida práctica a lo largo de los tiempos como una de las formas más privilegiadas de diálogo entre culturas. Pese a ello, es una actividad que se ha resistido, y se resiste todavía -aun siendo considerables los avances experimentados por la teoría y la práctica de las diversas modalidades de traducción-, a la sistematización y a verse reducida a una serie de preceptos que puedan ser reunidos en forma de tratado, por lo que son pocos los acercamientos a la traducción poética desde el lado de la práctica. Con todo, contamos con las breves explicaciones que algunos traductores muchas veces, ellos mismos poetas- han ido incluyendo en los prólogos a sus trabajos o en otros paratextos y en los que nos ofrecen sus reflexiones sobre la naturaleza y los límites de su experiencia traductora.

Lo que está fuera de toda duda es que la aspiración fundamental que debe guiar toda traducción poética es la reconstrucción o recreación integral en la lengua de llegada del texto originario. Como bien dice Teodoro Sáez Hermosilla: «[...] la traducción poética debe seguir entendiéndose como un intento de acercamiento a los textos originales mediante la reinvención de otros textos portadores, en la lengua término, de una "máxima” semejanza»².

Ello implica que no solo hay que intentar trasladar la información semántica, sino que hay que vigilar especialmente los rasgos métricos y formales, así como tener bien presente el nivel pragmático-estilístico y tratar de que el impacto producido por la traducción sea lo más análogo posible al del original. En otras palabras, podemos decir que la traducción de la poesía requiere unas estrategias propias y que debe tener en consideración tres aspectos:

- Una dimensión comunicativa que atiende a la traslación de unos significados.

- Un segundo nivel, en el que se persigue la transposición de una información estética de un modo lo más equivalente posible al que presenta el texto de partida.

- Y, por último, una fase en la que se busca la reexpresión de un sentido más allá de la simple combinación del plano del contenido y del plano de la expresión.

1. Pueden citarse, a título meramente de ejemplo, desde las palabras de Dante contenidas en el Convivio: "E però sappia ciascuno che nulla cosa per legame musaico armonizzata si può da la sua loquela in altra transmutare sanza rompere tutta sua dolcezza e armonia» (Convivio I, VII, 14) hasta el célebre aforismo de Robert Frost según el cual «Poetry is what gets lost in translation». La autenticidad y autoría de esta máxima no ofrecen ninguna duda, a pesar de no figurar en ninguno de los ensayos publicados del autor (cf. Mark Richardson, The Collected Prose of Robert Frost, Cambridge, MA, Harvard University Press, 2008).

2. Teodoro Sáez Hermosilla, La traducción poética a prueba: exégesis y autocrítica (ámbito francés-español), León, Secretariado de Publicaciones de la Universidad de León y Ediciones Universidad de Salamanca, 1998, p. 66. 
De ello se deduce que para llevar a buen fin esta labor son necesarias tres clases de habilidades: una competencia lingüística de los dos idiomas implicados para abordar el primer paso; una competencia literaria, compuesta, esencialmente, de amplios conocimientos filológicos y culturales, así como de determinadas aptitudes relacionadas con el funcionamiento de los textos poéticos; $y$, en tercer lugar, una competencia creativa que, trascendiendo el significado del texto y su aspecto formal, consiga reproducir las funciones y los efectos del poema originario. Como bien lo expone Amelia Gamoneda:

En la traducción de poesía está pues implicado un conocedor de la lengua de origen y de destino [...], un crítico literario para el análisis de las cualidades físicas de la lengua dinamizadas por el sentido en el texto de origen y un poeta (para dinamizar dichas cualidades en el texto de destino) ${ }^{3}$.

Estas tres figuras (el lingüista, el filólogo y el poeta o, cuando menos un amante de la poesía que tenga un alto sentido de la música, del lenguaje y del ritmo) suelen coincidir en una sola persona, por ser habitualmente la traducción poética un trabajo solitario y en solitario. Pero la gran complejidad de la empresa hace también posible que las distintas tareas en las que se descompone se distribuyan entre varias personas que unan sus esfuerzos en una estrecha colaboración. Colaboración, que no simple reparto, ya que, volviendo de nuevo a Amelia Gamoneda, en la traducción de poesía a varias manos: «Hay que repartir y compartir; y sobre lo que resulte, volver a repartir y compartir, y así llevarlo y traerlo procurando que no se caiga mucha materia prima con tanto traslado»"

De esta manera, al proceder tradicional de afrontar la traslación de un texto poético de forma individual ha venido a sumarse, en las últimas décadas, una modalidad que se ha mostrado particularmente satisfactoria, como es la traducción colectiva. El poeta y traductor brasileño Haroldo de Campos, al que debemos las versiones portuguesas de obras de autores tan relevantes como Dante, Goethe o Mallarmé, justifica, en cierto modo, esa práctica en su libro Metalinguagem. Ensaios de teoria e crítica literária, publicado en 1967. Dice así:

El problema de la traducción creativa sólo se resuelve en casos ideales, a mi ver, mediante un trabajo en equipo que agrupe, con la vista puesta en un objetivo común, a lingüistas y a poetas iniciados en la lengua de la que se va a traducir. Es necesario que la barrera entre artistas y profesores de lengua sea sustituida por una cooperación fértil, pero para eso es preciso que el artista (poeta o prosista) tenga de la traducción una idea correcta, como labor altamente especializada que exige una dedicación amorosa y pertinaz, y que, por su parte, el profesor de lengua posea lo que Eliot llamó «ojo creativo", es decir, que no esté limitado por prejuicios académicos, y que encuentre en la colaboración para la recreación de una obra de arte verbal aquel júbilo peculiar que procede de una belleza no para la contemplación, sino de una belleza para la acción o en acción ${ }^{5}$.

3. Amelia Gamoneda, «La lengua bífida de la traducción», en Javier Gómez Montero (ed.), Nuevas pautas de traducción literaria, Madrid, Visor, 2008, p. 39.

4. Op. cit., p. 65-66.

5. Haroldo de Campos, "Da traduçấo como critiçấo e como crítica», en Metalinguagem. 
Antes de esa fecha, no obstante, disponemos ya de importantes precedentes de traducciones literarias realizadas colectivamente: así, Joyce colaboró con el equipo de traducción de su Finnegan's Wake al francés y al italiano y Octavio Paz contó en sus traducciones con distintos colaboradores según las lenguas (el japonés, el chino o el sueco). Sin embargo, la primera experiencia de traducción colectiva organizada no se llevaría a cabo hasta 1983 en el Centre littéraire de la Fondation Royaumont (más tarde, Centre de Poésie \&Traduction), sito en la abadía del mismo nombre, a unos treinta quilómetros al norte de París. Efectivamente, una de las actividades principales -si no la más importantede este centro, dirigido por el poeta Bernard Noël, desde su fundación hasta su desaparición en el año 2000, y en el que colaboraron, entre otros, Rémy Hourcade y Emmanuel Hocquard, fue la organización de más de una cincuentena de seminarios de traducción poética colectiva que contaron con la presencia de los propios creadores. Valga recordar que de estos encuentros existen diversos textos editados por separado, así como una extensa antología titulada À Royaumont traduction collective 1983-2000 : une anthologie de poésie contemporaine (Grâne, Éditions Créaphis, 2000).

Posteriormente, algunos de los autores que tuvieron la ocasión de participar en esos seminarios quisieron introducir y reproducir esa experiencia en sus países de origen. De ese modo, a partir de 1990 se constituyó una red europea de centros de traducción de poesía contemporánea, situados en Alemania, España, Francia, Irlanda, Italia, Portugal, Rumanía, Turquía y Suecia, que fueron adoptando y adaptando el método de trabajo elaborado en Royaumont. En el ámbito español existen diversas experiencias, entre las que cabe destacar los Seminaris de Traducció Poètica de la Institució de les Lletres Catalanes en Farrera de Pallars, que se vienen celebrando con regularidad desde 1998; la Casa del Traductor de Tarazona, que organiza encuentros desde principios de la década de 1990 y que, desde 1999, forma parte del RÉCIT (Réseau Européen des Centres de Traducteurs Littéraires); y, ya más recientemente, el Círculo de Traducción Poética de Córdoba, que empezó su andadura en 2006.

A ellos hay que añadir el Taller de Traducción Literaria de la Universidad de La Laguna (TTL), del que formo parte, creado en junio de 1995 por iniciativa de Andrés Sánchez Robayna, tan solo unos meses después de haber tenido la oportunidad de conocer muy de cerca como poeta la experiencia de Royaumont. Tal y como se ha explicado en distintas ocasiones ${ }^{6}$, el principal objetivo de este grupo de trabajo es el de estudiar las cuestiones y los problemas de los que se

Ensaios de teoria e crítica literária, São Paulo, Petrópolis Vozes, 1967. Citamos por la traducción española de Rodolfo Mata: «De la traducción como creación y como crítica», en De la razón antropofágica y otros ensayos, México, Siglo XXI, 2000, p. 201.

6. Andrés Sánchez Robayna, «El Taller de Traducción Literaria», Diario 16, supl. «Culturas», 23 de noviembre 1996; Alejandro Rodríguez Refojo, «Diez años del Taller de Traducción Literaria de la Universidad de La Laguna», Piedra y Cielo, no 3, 2005, p. 22-28; Andrés Sánchez Robayna, "Acerca del Taller de Traducción Literaria», Insula, n 717, 2006, p. 2-4; Andrés Sánchez Robayna, «Introducción», en Andrés Sánchez Robayna (ed.), De Keats a Bonnefoy. Versiones de poesía moderna, Valencia, Pre-Textos, 2006, p. 11-22. 
ocupa la llamada Traductología, de una parte, y, de otra, llevar a cabo diferentes trabajos prácticos de traducción. En contraposición con los demás equipos que se han señalado, en el TTL de La Laguna no siempre se traducen poetas contemporáneos y, cuando se da el caso, se cuenta muy excepcionalmente con la presencia del autor traducido. Por otra parte, y a diferencia de las experiencias de Royaumont y de Tarazona, se trata de un grupo estable y abierto que se reúne de forma regular. En efecto, desde su fundación, sus integrantes, entre los que se encuentran tanto profesores como otras personas amantes de la traducción literaria y, esporádicamente, algunos estudiantes, se dan cita todos los martes por la tarde entre octubre y junio, siguiendo unas mínimas normas de funcionamiento y unos claros criterios de traducción ${ }^{7}$.

Siempre con miras a reconstruir en español de la manera más equivalente posible la información semántica, estética y pragmático-estilística contenida en los textos de partida, en el seno del TTL se emplean dos grandes sistemas. El primero que se llevó a la práctica, y que se sigue utilizando en casos puntuales, es la traducción colectiva propiamente dicha, que fue especialmente fructífera durante los ańos iniciales del seminario ${ }^{8}$. Este procedimiento empieza siempre por una lectura del poema original, tras la cual se lleva a cabo un análisis textual que saque a la luz los rasgos más relevantes de la obra desde el punto de vista semántico, gráfico, sonoro, rítmico, etc., así como las previsibles dificultades de interpretación. A continuación, se inicia la traducción verso a verso y estrofa a estrofa con la ayuda de todos los participantes y de las herramientas disponibles (diccionarios bilingües y monolingües, ideológicos, de sinónimos y de dudas, versiones del texto en otras lenguas, etc.). Se intercambian opiniones, se discuten las distintas soluciones que se van proponiendo y se elige (con votación, si es preciso) la propuesta que la mayoría considere más ajustada. Paralelamente, se van realizando las lecturas parciales que sean necesarias, tanto de los versos de partida como de los traducidos. Por último, se lee el resultado final al completo y, si se da el caso, se revisan o reconsideran algunas de las decisiones tomadas. Con posterioridad a las sesiones presenciales, y ya de manera individual, se llevan a cabo las lecturas o retoques que se juzguen oportunos, que deben contar, lógicamente, con el visto bueno de los demás integrantes del grupo.

El segundo de los métodos que se siguen, el de la revisión colectiva, parte de una traducción individual previa realizada por uno de los participantes en el seminario. Este, como es evidente, no solo debe poseer un profundo conocimiento de la lengua de partida -en la mayoría de los casos, se trata de hablantes nativos de esa lengua o de especialistas en su filología- y una gran capacidad expresiva en español, sino también una "competencia de

7. Con el propósito de divulgar la labor realizada y los proyectos en curso, así como de publicar escritos críticos relacionados con la traducción literaria, en otońo de 2011 se publicó el primer número del Boletín del Taller de Traducción Literaria de la Universidad de La Laguna, cuya segunda entrega ha visto la luz en febrero de 2012.

8. Con este proceder, recordemos que solo en 1996 se publicaron, en las ediciones del propio centro, cuatro obras: Paolo Valesio, Sonetos profanos y sacros; Keats, Oda a una urna griega; Jacques Ancet, La habitación vacía y Claude Esteban, Siete días de ayer. 
transferencia», como así la llama Amparo Hurtado", aludiendo a la capacidad de descodificar un texto en una lengua y recodificarlo en otra. El traductor presenta y defiende su propuesta, habitualmente una versión casi literal que puede contemplar distintas alternativas o variantes, y en la que prima la búsqueda de la correspondencia de significado, sin ignorar factores de carácter pragmático como el registro de lengua, la frecuencia de uso o las marcas estilísticas, al tiempo que va comentando los problemas que presenta el texto. En algún caso, sin embargo, este borrador puede ser más cuidado y preocuparse también de la traslación de la información estética. Como paso siguiente, esta propuesta provisional es revisada de forma conjunta por el resto de los miembros del grupo, los cuales, sirviéndose de los mismos criterios y de las mismas herramientas que se señalaban para la traducción colectiva pura, le sugieren alternativas, modificaciones o correcciones, que el traductor suele aceptar (aunque puede no hacerlo al ser el firmante de la versión definitiva). Con este sistema, más efectivo y rápido que el anterior, se han publicado, tanto en las ediciones del propio TTL como en distintas editoriales españolas, más de quince títulos de autores franceses, ingleses, italianos, catalanes, portugueses, alemanes y griegos ${ }^{10}$.

En cualquier caso, sea cual sea la fórmula aplicada, los participantes deben estar dispuestos a dialogar, pactar, rectificar, retocar y pulir, o cuando menos, a respetar el punto de vista de los demás. Y, por regla general, en un momento dado del proceso se alcanza ese punto mágico que Rémy Hourcade, refiriéndose a su propia experiencia en Royaumont, llamó «el traductor colectivo» ${ }^{11}$, que se

9. Amparo Hurtado Albir, «Objetivos de aprendizaje y metodología en la formación de traductores e intérpretes", en Enseñar a traducir. Metodología en la formación de traductores e intérpretes, Madrid, Edelsa, 1999, p. 43.

10. Gustave Flaubert, Diccionario de lugares comunes (trad. Fátima Sainz y Ángel Mollá, La Laguna, Ediciones Canarias, 1997); Bernard Noël, El sindrome de Gramsci (trad. Guy Rochel, La Laguna, Ediciones Canarias, 1998); William Wordsworth, El preludio (trad. Fernando Galván y Andrés Sánchez Robayna, La Laguna, Ediciones Canarias, 1999); Samuel Johnson, Prefacio a Shakespeare (trad. Carmen Toledano, Barcelona, Acantilado, 2003); Geoffrey Hill, Veintisiete poemas (trad. Jordi Doce, Tegueste, Taller de Traducción Literaria, 2003); Edmond Jabès, Un extranjero con, bajo el brazo, un libro de pequeño formato (trad. Cristina G. de Uriarte y Maryse Privat, Barcelona, Galaxia Gutenberg, 2002); Wallace Stevens, De la simple existencia (trad. Andrés Sánchez Robayna, Barcelona, Galaxia Gutenberg, 2003); Maria Mercè Marçal, Deshielo (trad. Clara Curell, Montblanc, Igitur, 2004) Andrés Sánchez Robayna (ed.), De Keats a Bonnefoy Versiones de poesía moderna. Diez años del Taller de Traducción Literaria (Valencia, Pre-Textos, 2006); Paul Valéry, Cuadernos (trad. Maryse Privat, Andrés Sánchez Robayna y Fátima Sainz, Barcelona, Galaxia Gutenberg, 2007); Jaume Pont, Vuelo de cenizas (trad. Clara Curell, Valencia, Pre-Textos, 2008); Fabrizio dall'Aglio, Hic et Nunc (trad. Sarah Pelusi, Madrid, Huerga y Fierro, 2008); Giorgos Seferis, Tres poemas secretos (trad. Isabel García Gálvez, Madrid, Abada Editores, 2009); Mario Luzi, Antología poética (trad. Jesús Díaz Armas, Barcelona, Galaxia Gutenberg I Círculo de Lectores, 2009); Andrés Sánchez Robayna (ed.), Ars poetica. Versiones de poesía moderna. (Quince años del Taller de Traducción Literaria) (Valencia, Pre-Textos, 2011); Hart Crane, El puente (trad. Sally Burgess y Margarita Fernández de Sevilla, Valencia, Pre-Textos, en prensa).

11. Rémy Hourcade, «La experiencia de Royaumont», en P. Valesio y R.-J. Díaz, Literatura y traducción: caminos actuales, Santa Cruz de Tenerife, Universidad Menéndez Pelayo, 1996, p. 156. 
produce cuando el grupo se pone a hablar con una sola voz. Otra de las buenas costumbres del TTL es que a lo largo de todos los seminarios se van anotando las distintas variantes surgidas y las versiones intermedias, lo que permite en cualquier momento rastrear el proceso de toma de decisiones y analizar los diferentes estadios de la labor realizada ${ }^{12}$.

Por lo que atañe ya a los criterios que se aplican en la actividad traductora propiamente dicha, hay que decir que se cuidan con especial atención los aspectos rítmicos y métricos, respetándose de manera rigurosa el número de versos y, en su caso, de estrofas del poema de partida, así como adaptando, del modo más equivalente posible, el esquema silábico y acentual originario. En especial, por lo que concierne a la métrica, se suele acudir a las formas impares, más acordes con la tradición española, mientras que en lo que respecta a la rima, el criterio general es prescindir de ella a favor del verso blanco ${ }^{13}$.

A lo largo de la extensa andadura del Taller de Traducción Literaria de La Laguna, creo no equivocarme al afirmar que todos sus miembros estamos convencidos de que la labor en equipo supone un enriquecimiento y una garantía de trabajo bien hecho. En efecto, conscientes, como bien lo apunta Yves Bonnefoy, de que «interpréter est une suite sans fin de décisions parfois hasardeuses» ${ }^{14}$, hemos podido comprobar cómo esos riesgos pueden reducirse gracias a la detenida labor de confrontación, de intercambio, de discusión y de revisión a la que sometemos todos nuestros acuerdos.

Como ilustración de lo dicho hasta ahora, me ha parecido interesante traer aquí, a simple modo de ejemplo, dos traducciones realizadas recientemente en el seno de nuestro taller siguiendo el procedimiento de traducción revisada y que están incluidas en el volumen Ars poetica. Versiones de poesía moderna. (Quince años del Taller de Traducción Literaria) que acaba de ver la luz hace unos meses $^{15}$. En ambos casos ofrezco aquí el texto original, la versión española final, así como una propuesta previa, para, de este modo, poder seguir, aunque sea a grandes trazos, las distintas fases del proceso traslativo.

La primera es la versión (realizada por Estela L. Jiménez Marzán y el TTL) del poema «Naissance à l'orage» de Pierre Reverdy, que forma parte de la antología Plupart du temps. 1915-1922 (París, Gallimard, 1945).

12. Ello ha permitido realizar varios estudios, además del presente, que abordan el sistema de trabajo seguido por el TTL, como el de Sally Burgess y Carmen Toledano centrado en la traducción de poemas de Conrad Aiken y Hart Crane (cf. bibliografía final).

13. El mantenimiento de la rima conduce en muchos casos a construcciones forzadas e, incluso, a sacrificar el contenido del original.

14. Yves Bonnefoy, La communauté des traducteurs, Estrasburgo, Presses Universitaires de Strasbourg, 2000, p. 13.

15. Incluye textos originales y traducciones españolas de 35 autores, de William Wordsworth a Adam Zagajewski, realizadas entre 2006 y 2010. 

1 Toute la face ronde
Au coin sombre du ciel
L'épée
5 sous les rideaux de l'air
la mappemonde

NAISSANCE À L'ORAGE

Dans la chambre à l'envers

Des paupières plus longues

Un nuage s'effondre

La nuit sort d'un éclair

Como se puede comprobar, el poema de Reverdy es una breve pieza de ocho versos hexasílabos con una singular disposición gráfica, escrita con un léxico simple y bastante usual y una sintaxis sin dificultades importantes, a base de frases nominales o verbales breves.

La versión de partida sobre la que trabajamos fue una traducción literal, resultado de una simple traslación semasiológica sin ninguna pretensión de entrar en otras dimensiones, que serían abordadas posteriormente entre todos, salvo el aspecto visual, que fue escrupulosamente respetado desde el principio ${ }^{16}$.

\author{
[primera versión] \\ NACIMIENTO DE LA TORMENTA \\ 1 Toda la cara redonda \\ En el rincón sombrio del cielo \\ La espada \\ 5 \\ el mapamundi \\ bajo las cortinas del aire \\ En la habitación al revés \\ Unos párpados más largos \\ Una nube se hunde \\ La noche aparece de un relámpago
}

Uno de los primeros escollos con los que tropezamos en las sesiones conjuntas fue la traducción del título, en particular, la de la preposición à. Para algunos de los participantes, la preposición española de cubría los posibles sentidos del francés, sobre todo si se consideraba que el significado principal aludía al resultado de (donner) naissance: la tormenta era la que había nacido. Para otros, si el sintagma equivalía efectivamente a "dar nacimiento" a algo, con elipsis del verbo, la preposición a podría igualmente ser válida, aunque

16. Se resaltan en cursiva aquellos elementos que plantearon algún problema o suscitaron un mayor debate. 
producía una secuencia poco habitual en español: "nacimiento $a$ la tormenta». Otra posibilidad barajada fue interpretar la locución preposicional como un locativo: «en la tormenta». Sin embargo, la solución mayoritaria fue decantarse por la preposición con, entendiendo que el nacimiento se producía 'junto a' o ‘junto con' la tormenta.

En cuanto al patrón métrico, su elección no planteó discusión alguna, toda vez que es criterio habitual en el grupo, como ya se apuntó antes, recurrir a la medida impar más equivalente al original. En este caso, el hexasílabo francés se convirtió en un pie de siete sílabas, lo que supuso no pocos ajustes léxicos con respecto a la primera versión: así, cara (v. 1) se sustituyó por su sinónimo faz, voz etimológicamente más cercana al francés, pero perteneciente a un registro más elevado y de uso poco corriente; el rincón sombrio (v. 2) pasó a ser simplemente lo oscuro, puesto que la necesaria reducción silábica obligó a omitir el elemento rincón, cuya pérdida nos pareció semánticamente poco relevante al no constituir una palabra clave en el conjunto del poema; las cortinas (v. 5) se transformaron en un único telón y la habitación (v. 7) en su equivalente cuarto. La sustitución de aparece (v. 9) por surge, además de obedecer a razones métricas, se debió a su mayor proximidad semántica con el verbo francés y a su relación con la idea de nacimiento que preside el poema.

Otras decisiones se refirieron al plano morfosintáctico, como la transposición del artículo indefinido des (v. 6) por el definido los, o la inversión del orden de los elementos en el octavo verso (se desploma una nube) por motivos no solo de cómputo silábico, sino también de índole rítmica y eufónica.

De este modo, la versión final quedó como sigue:

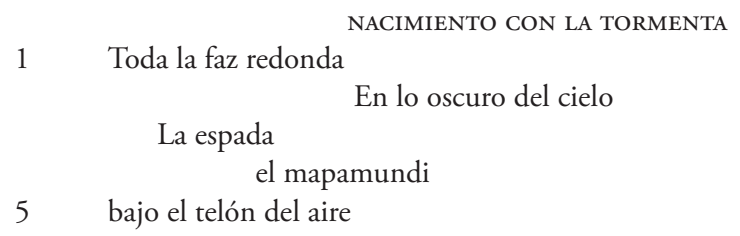

En el cuarto al revés

Los párpados más largos

Se desploma una nube

La noche surge de un relámpago

El segundo ejemplo que presento es mi traducción de la composición «Illa» de Josep Carner, publicada en 1952 en el poemario Llunyania (Santiago de Chile, El Pi de les Tres Branques; edición definitiva en Poesia, Barcelona, editorial Selecta, 1957). He aquí el original:

\section{ILLA}

1 Oh penyalar sobre camins que dansen,

illa, sobtada solitud, prodigi,

castell en mar, que mira, fonedissos, 
el núvol, el vaixell! No pas que et manqui ni mai no l'aturis, el rosec dels dies. En tos covals, les ones fan esquerda, el braç Cap-a-la-Terra se t'escurça, $i$ els teus pins s'escabellen, temorosos de l'ahuc de les mòbils fondalades.

10 Tu i jo dempeus! I baldament s'escaigui que juguem algun cop a estrangeria (hàbit com és que fins l'amor destrii), tu i jo plegats! Des la naixença meva que vetlles el meu bleix; tu m'inventares

15 formes, colors, perquè em vagués de viure. I en veient-me descloure les parpelles, goses, cada mati, de viure encara. No tingués jo matins, ¿qui et refaria?

Els meus sentits, d'encantament emplenes: el vent, vestit de pols, brinat d'escuma, el cel, amb el folcat que hi fa rodona, el vell Proteu, musicador dels canvis, la vida, pressa d'alenades vanes, el foll delit que dins la sang arboren 25 les soles lluentors de la parença $i$ la virtut, arreu desassistida, provant l'espai amb el seu angle d'ales.

Tot és senyal, i cap senyal no dura. ¿Què mai podries tu, penya vermella, 30 dins de la grapa universal del trànsit, ni que fos ver el teu posat de roca, certitud de pendissos i d'arestes; ni que no fossis illa imaginària, feta, refeta $i$ habitada en somnis, 35 bastida amb el record i l'impossible, sols al meu esperit commensurable?

Illa tres voltes! Perquè tens un ròdol enllà de tot, que em crida i no em contesta, $i$ un altre, fet de comitives d'ombres,

$40 \quad i$ un, a tocar, de boires i complantes. I ja mos ulls sobliden de la terra, $i$ la ressaca entre mos dits sescola. I el tot enyoro que en la llum traspunta, del mig estant d'una insabuda cala,

45 centre d'un arc que em protegeix i em lliura.

Que avui el sol, quan davant meu davalli, em trobi encara a punt per a guarnir-me un focarret, ull tendre de la fosca, última feina d'horabaixes. -Illa, Oh si poguessis, ja mai més feixuga, 


\author{
com un vaixell vogar per les tenebres, \\ sense indici de solc en el silenci, \\ els pals guerxats, però la vela viva!
}

En este caso, la versión intermedia que les ofrezco no es un primer borrador, es decir, una traducción literal y plana, sino una propuesta más avanzada, bastante elaborada y medida, con varias alternativas, en la que no solo intenté trasladar el plano semántico, sino que me preocupé asimismo de la sustancia estética. $\mathrm{Al}$ ser una muestra mucho más extensa que la anterior, un poema de seis estrofas compuestas por nueve versos decasílabos, solamente me detendré en comentar, en primer lugar, algunos de los recursos que empleé para tratar de encontrar la máxima equivalencia y adecuarme al esquema silábico y rítmico del español y, a continuación, aquellos aspectos que se mejoraron de forma sustancial en la versión final gracias al trabajo de revisión colectiva.

\title{
[versión provisional]
}

IsLA

¡Oh riscos sobre sendas danzarinas [que retozan], isla, soledad súbita, prodigio, castillo en mar, que ve desvanecerse [que mira, evanescentes], el navío, la nube! No te falta [Nunca falta], ni jamás lo detengas [ni lo descartes nunca], el roce [el paso/ la erosión] de los días.

En tus grutas, las olas abren grietas, tu brazo [istmo] hacia la tierra va mermando, tus pinos se despeinan [desgreńan], temerosos [aterrados] del aullido [por el aullar] de tus abismos móviles.

$10 \quad$ iEn pie tú y yo! Por mucho que finjamos alguna vez que no nos conocemos [que somos extranjeros] (es habitual que hasta el amor separe [no es raro que el amor hasta separe]), juntos tú y yo! Vigilas mis latidos desde mi nacimiento; y para mí creaste las formas, los colores, que alientan [animan/ impulsan] mi vivir. Y al ver que cada día abro los párpados [los ojos], te atreves a seguir con tu existencia. Sin mis mañanas, ¿quién te reharía?

Mis sentidos inundas de embeleso: el viento, con su traje hecho de espuma y polvo, el cielo y su manada [rebaño] que circula [que da vueltas], Proteo, el viejo mago, hacedor de los cambios, la vida, impaciencia de inútiles afanes [alientos/impulsos], el delirio insensato que en la sangre sólo enciende el fulgor de la apariencia y la virtud, que siempre sin amparo, va probando el espacio con su ángulo de alas.

Todo es señal, y no hay señal que dure. ¿Podrás acaso tú, roque [risco] encarnado [bermejo], atrapado en el tránsito absoluto, 
aunque fuera real [verdad] tu apariencia de roca, evidencia de cuestas y de aristas; aunque no fueras isla imaginaria, hecha, rehecha y habitada en sueños, construida de [con] recuerdos e imposibles, sólo conmensurables en mi espíritu?

¡Isla en tres vueltas! Tienes un círculo remoto que de lejos me llama y no contesta, otro está hecho de sombras que desfilan, y el otro, más cercano, de brumas y lamentos. Ya mis ojos se olvidan de la tierra, y la resaca fluye entre mis dedos. Y ańoro todo aquello que despunta en la luz, que veo desde el fondo de de una cala ignorada, eje a la vez de un arco que me ampara y me entrega

Que hoy cuando el sol descienda ante mi vista, me encuentre listo aún para prender un fuego, ojo sensible de lo oscuro, la última labor de los ocasos. -Isla, ¿y cuándo [cuándo pues] dormiremos el sueño irrevocable? ¡Oh si pudieras, ya libre de peso [carga], como un barco bogar [remar] por las [entre] tinieblas, sin indicios [señales] de surco en el silencio, con mástiles [los mástiles] combados [torcidos], pero la vela viva!

En lo referente a los procedimientos aplicados, señalaré para empezar una serie de transposiciones, como la utilizada en el primer verso, mediante la cual la oración de relativo camins que dansen pasó a ser el sintagma nominal sendas danzarinas; la transformación, en el verso 7 , de la forma verbal simple se téscurça en la forma perifrástica va mermando o, también, la sustitución del sintagma adjetival arreu desassistida (v. 26) por el sintagma nominal siempre sin amparo. En otros casos, tuve que acudir a la modificación del número del sustantivo o sintagma original y, de esta forma, el penyalar del primer verso se convirtió en riscos y el record $i$ l'impossible del verso 35 dieron en el texto de llegada la forma plural recuerdos e imposibles. En algún otro momento, razones métricas y acentuales no hicieron posible emplear el equivalente castellano exacto, por lo que se usó un sinónimo contextual, con el inevitable menoscabo semántico que ello supone, como sucedió en el verso 22 al traducir el vell Proteu, musicador dels canvis por Proteo, el viejo mago de los cambios. En otra ocasión me valí de una técnica de traducción oblicua más extrema, como es la explicitación, $\mathrm{y}$, así, el enunciado original juguem algun cop a estrangeria dio lugar a una cláusula más expresa tanto en la versión provisional, finjamos alguna vezl que somos extranjeros, como en la versión final finjamos alguna vez/ que somos como extraños (v. 10-11).

Otras veces, las necesidades de cadencia o cómputo silábico obligaron a realizar modificaciones en el plano morfosintáctico y, de ese modo, el orden de las palabras se alteró o invirtió en no pocos versos, como en el v. 2 (sobtada 
solitud > soledad súbita), en el v. 4 (el núvol, el vaixell > el navio, la nube), en el v. 10 (tu i jo dempeus > en pie tú y yo) o en el v. 24 (el foll delit > el delirio insensato).

En cuanto a las revisiones colectivas que dieron lugar a la versión definitiva, estas consistieron en su mayoría en tratar de convertir algunos alejandrinos en endecasílabos, medida mucho más equivalente al decasílabo del poema original. Es el caso, por ejemplo, del v. 5 en el que la propuesta provisional múltiple $n i$ jamás lo detengas [ni lo descartes nunca,], el roce [el pasol la erosión] de los dias quedó, finalmente, en ni pares nunca, el roce de los dias; lo mismo ocurrió en el v. 20, en el que el viento, con su traje hecho de espuma y polvo se redujo a el viento, con disfraz de espuma y polvo o, también, en el v. 31, al transformar aunque fuera real tu apariencia de roca en aunque fuera verdad tu aire de roca, a mi modo de ver, un verdadero hallazgo. Sin embargo, las indiscutibles habilidades del grupo no consiguieron, por su extrema dificultad, reproducir la polisemia del sintagma illa tres voltes (v. 37), esto es, 'isla tres veces', pero también, de forma más velada, 'isla en tres vueltas', que en la primera propuesta se reducía a isla en tres vueltas y que, finalmente, quedó como tres veces isla.

La versión final es la siguiente:

IsLA

$¡$ Oh riscos sobre sendas danzarinas, isla, soledad súbita, prodigio, castillo en mar, que mira, evanescentes, el navío, la nube! No te falta, ni pares nunca, el roce de los días. En tus grutas, las olas abren grietas, tu brazo hacia la tierra va mermando, tus pinos se despeinan, temerosos del aullar de las móviles honduras.

¡En pie tú y yo! Por mucho que finjamos alguna vez que somos como extraños (es habitual que aun el amor separe), juuntos tú y yo! Vigilas mis latidos ya desde que nací; formas, colores, me inventaste, que alientan mi vivir. Cada mañana, al verme abrir los párpados, te atreves a seguir con tu existencia. Sin mis mañanas, ¿quién te reharía?

Inundas mis sentidos de embeleso: el viento, con disfraz de espuma y polvo, el cielo y su rebaño que da vueltas, Proteo, el viejo mago de los cambios, la vida, apremio del aliento inútil, el delirio insensato que en la sangre sólo enciende el fulgor de la apariencia y la virtud que, siempre sin amparo, prueba el aire con su ángulo de alas. 
Todo es señal, y no hay señal que dure. ¿Acaso puedes tú, risco bermejo, en el encierro universal del tránsito, aunque fuera verdad tu aire de roca, evidencia de cuestas y de aristas; aunque no fueras isla imaginaria, hecha, rehecha y habitada en sueños, alzada con recuerdos e imposibles, en mi espíritu sólo mensurables?

Tres veces isla: un apartado círculo que de lejos me llama y no contesta, otro, hecho de sombras que desfilan, y otro, más cerca, de lamento y brumas. Ya mis ojos se olvidan de la tierra, y la resaca fluye entre mis dedos. Y añoro el todo que en la luz despunta, contemplado desde una cala ignota, centro de un arco que me ampara y libra.

Que hoy cuando el sol descienda ante mi vista, me encuentre listo aún para prender un fuego, ojo sensible de lo oscuro, labor final de los ocasos. - Isla, ¿cuándo dormir el sueño irrevocable? $¡ O h$ si pudieras, libre ya de carga, como un barco bogar entre tinieblas, sin indicio de surco en el silencio, curvos los palos, mas la vela viva!

Estas breves explicaciones con las que he querido acercarme de manera sucinta al funcionamiento del Taller de Traducción Literaria de la Universidad de La Laguna no pueden reflejar, como es lógico, las muchas horas de esfuerzo que hay detrás de cada propuesta, el fructífero debate del que surge cada decisión y, lo que es más importante, el reto y el aprendizaje constante que para sus integrantes suponen todas y cada una de las sesiones de trabajo. En este sentido, las palabras de Pierre Leyris, uno de los traductores franceses más respetados de su generación ${ }^{17}$, marcan nuestro camino diario: «Traduire, c'est avoir l'honnêteté de s'en tenir à une imperfection allusive» ${ }^{18}$.

El haber superado ya los tres lustros, el reconocimiento a nuestra labor que han manifestado otros traductores y poetas, y, por último, el hecho de que sirva de modelo para otros grupos similares, como los encuentros de escritores

17. A él le se le debe la dirección, junto con Henri Evans, de la edición bilingüe en trece volúmenes de la obra completa de Shakespeare (París, Club Français du Livre, 1954-1961), así como la traducción de Eliot, Yeats, De Quincey o Milton, entre otros muchos autores anglófonos, lo que le valió en 1985 el Grand Prix National de la Traduction. En La Chambre du traducteur (José Corti, 2007) se incluyen varios de sus ensayos sobre su experiencia como lector $\mathrm{y}$ traductor de las letras anglosajonas.

18. Incluidas en una entrevista publicada en Le Monde el 12 de julio de 1974. 
y traductores de Castrillo de los Polvazares o el Taller de Traducción Literaria de Kiel, no hacen sino confirmar el acierto de esta experiencia de traducción polifónica.

\section{Bibliografía}

Burgess Sally, Toledano Carmen, «Explicitation in the collaborative translation into Spanish of two modern American poets», en Oleksy Wieslaw y Stalmaszczyk Piotr (eds.), Cognitive Approaches to Language and Linguistic Data, Frankfurt am Main, Peter Lang, p. 387-400.

Bonnefoy Yves, La communauté des traducteurs, Estrasburgo, Presses Universitaires de Strasbourg, 2000.

Campos Haroldo de, "Da tradução como critição e como crítica», en Metalinguagem. Ensaios de teoria e crítica literária, São Paulo, Petrópolis Vozes, 1967. [Traducción española de Rodolfo Mata: «De la traducción como creación y como crítica», en De la razón antropofágica y otros ensayos, México, Siglo XXI, 2000, p. 185-204].

Dante Alighieri, Convivio I, VII, Edizione U.T.E.T [en línea]. Disponible en: http://www.classicitaliani.it/dante/convivio/convivio_trattato_1.htm [consultado el 2 de octubre de 2011].

Gamoneda Amelia, «La lengua bífida de la traducción», en Gómez Montero Javier (ed.), Nuevas pautas de traducción literaria, Madrid, Visor, 2008, p. 37-73.

Hourcade Rémy, «La experiencia de Royaumont», en Valesio P. y Díaz R.-J., Literatura y traducción: caminos actuales, Santa Cruz de Tenerife, Universidad Internacional Menéndez Pelayo, 1996, p. 155-161.

Hurtado Albir Amparo, "Objetivos de aprendizaje y metodología en la formación de traductores e intérpretes», en Enseñar a traducir. Metodología en la formación de traductores e intérpretes, Madrid, Edelsa, 1999, p. 8-58.

Richardson Mark, The Collected Prose of Robert Frost, Cambridge, MA, Harvard University Press, 2008.

Sáez Hermosilla Teodoro, La traducción poética a prueba: exégesis y autocritica (ámbito francés-español), León, Secretariado de Publicaciones de la Universidad de León y Ediciones Universidad de Salamanca, 1998. 